\title{
Endothelial function does not improve with high-intensity continuous exercise training in SHR: implications of eNOS uncoupling
}

\begin{abstract}
Sylvain Battault ${ }^{1}$, François Singh ${ }^{2}$, Sandrine Gayrard ${ }^{1}$, Joffrey Zoll ${ }^{2}$, Cyril Reboul ${ }^{1,3}$ and Grégory Meyer ${ }^{1,3}$
Exercise training is a well-recognized way to improve vascular endothelial function by increasing nitric oxide (NO) bioavailability. However, in hypertensive subjects, unlike low- and moderate-intensity exercise training, the beneficial effects of continuous high-intensity exercise on endothelial function are not clear, and the underlying mechanisms remain unknown. The aim of this study was to investigate the impact of high-intensity exercise on vascular function, especially on the NO pathway, in spontaneous hypertensive rats (SHR). These effects were studied on WKY, sedentary SHR and SHR that exercised at moderate (SHR-MOD) and high intensity (SHR-HI) on a treadmill ( $1 \mathrm{~h}$ per day; 5 days per week for 6 weeks at $55 \%$ and $80 \%$ of their maximal aerobic velocity, respectively). Endothelial function and specific NO contributions to acetylcholine-mediated relaxation were evaluated by measuring the aortic ring isometric forces. Endothelial nitric oxide synthase (eNOS) expression and phosphorylation (ser1177) were evaluated by western blotting. The total aortic and eNOS-dependent reactive oxygen species (ROS) production was assessed using electron paramagnetic resonance in aortic tissue. Although the aortas of SHR-HI had increased eNOS levels without alteration of eNOS phosphorylation, high-intensity exercise had no beneficial effect on endothelium-dependent vasorelaxation, unlike moderate exercise. This result was associated with increased eNOS-dependent ROS production in the aortas of SHR-HI. Notably, the use of the recoupling agent $\mathrm{BH}_{4}$ or a thiol-reducing agent blunted eNOS-dependent ROS production in the aortas of SHR-HI. In conclusion, the lack of a positive effect of high-intensity exercise on endothelial function in SHR was mainly explained by redox-dependent eNOS uncoupling, resulting in a switch from $\mathrm{NO}$ to $\mathrm{O}_{2}^{-}$generation.
\end{abstract}

Hypertension Research (2016) 39, 70-78; doi:10.1038/hr.2015.114; published online 5 November 2015

Keywords: strenuous exercise; vascular function; nitric oxide

\section{INTRODUCTION}

Hypertension is a major public health problem, affecting approximately one billion people worldwide. ${ }^{1}$ In humans and in experimental rodent models, essential hypertension is often associated with impaired endothelium-dependent vasorelaxation, which contributes to the progression of the pathology $y^{2,3}$ and aggravates the cardiovascular risk. ${ }^{4-7}$ Based on these observations, several studies have aimed to evaluate the potential beneficial effects of improving endothelial function in hypertensive subjects, ${ }^{8,9}$ which has been shown to confer a more favorable prognosis. ${ }^{9}$

Exercise training has been largely recognized as an efficient strategy in the management of endothelium-dependent vascular impairment in humans and in experimental models of essential hypertension. ${ }^{10}$ Indeed, exercise training improves endotheliumdependent vasodilatory capacity ${ }^{11-13}$ primarily by improving nitric oxide (NO) bioavailability. Current evidence shows that exercise training is associated with increased eNOS expression, ${ }^{14,15}$ phosphorylation of its main activation site $(\operatorname{ser} 1177)^{16}$ and stabilization of its dimerized state. ${ }^{17,18}$ In addition, exercise training increases antioxidative enzyme activity, which blunts reactive oxygen species (ROS) production ${ }^{19}$ and contributes to prevent the reaction between ROS and $\mathrm{NO},{ }^{20,21}$ rendering NO more available.

However, the benefits of exercise on endothelial function depend on the exercise intensity. Although high-intensity $(\geqslant 80 \%$ of the maximal aerobic velocity) exercise training has been consistently reported to improve endothelium, and more specifically NO-dependent vasorelaxation, in normotensive subjects, ${ }^{22-24}$ this positive effect has not been reported in hypertensive models. ${ }^{25}$ Moreover, the underlying mechanisms remain unknown and whether the endothelial nitric

${ }^{1}$ Laboratoire de Pharm-Ecologie Cardiovasculaire (EA4278), Faculty of Sciences, Avignon University, Avignon, France and ${ }^{2}$ E.A. 3072, Fédération de Médecine Translationnelle, Faculty of Medicine, University of Strasbourg, Strasbourg, France

${ }^{3}$ These two authors are senior co-authors.

Correspondence: Dr C Reboul, Laboratoire de Pharm-Ecologie Cardiovasculaire (EA4278), Faculty of Sciences, Avignon University, 33 rue Louis Pasteur, Avignon 84000, France. E-mail: cyril.reboul@univ-avignon.fr

or Dr G Meyer, Laboratoire de Pharm-Ecologie Cardiovasculaire (EA4278), Faculty of Sciences, Avignon University, 33 rue Louis Pasteur, Avignon 84000, France.

E-mail: gregory.meyer@univ-avignon.fr

Received 27 May 2015; revised 30 July 2015; accepted 20 August 2015; published online 5 November 2015 
oxide synthase (eNOS)-NO pathway could be negatively affected remains to be investigated.

The aim of this study was to evaluate the consequence of continuous high-intensity exercise training $(80 \%$ of the maximal aerobic velocity, $1 \mathrm{~h}$ per day for 6 weeks) on the endothelial function and eNOS functional state in spontaneous hypertensive rats (SHR). As expected, unlike moderate-intensity exercise, continuous highintensity exercise training did not enhance endothelial function in this model. This lack of a beneficial effect of continuous high-intensity exercise seems to be mainly explained by a redox-dependent uncoupling of eNOS, reinforcing ROS production in the aortic tissue of SHR. ${ }^{26}$

\section{METHODS}

\section{Experimental protocol}

All investigations complied with the Guide for the Care and Use of Laboratory Animals published by the US National Institutes of Health (NIH Publication No. 85-23, revised 1996) and with the regulations of the French Ministry of Agriculture. All experiments were approved by the ethics committee of Marseille (CNREEA 14). Male SHR $(n=24)$ and Wistar Kyoto (WKY) rats $(n=10)$ were obtained from Janvier (Laval, France) at 10 weeks of age. Four rats were housed per cage under controlled conditions of temperature $\left(21^{\circ}\right.$ $\mathrm{C} \pm 1)$, humidity $(60 \% \pm 10)$ and lighting ( $12 \mathrm{~h}$ a day). They had free access to tap water and standard food. After a period of acclimation, the maximal aerobic velocity of SHR was evaluated using a driven treadmill during a continuous and progressive maximal exercise test. The driven treadmill was set at a speed of $10 \mathrm{~m} \mathrm{~min}^{-1}$ for $3 \mathrm{~min}$, after which the speed was increased by $3 \mathrm{~m} \mathrm{~min}^{-1}$ every $90 \mathrm{~s}$ until $85-90 \%$ of the expected maximal aerobic velocity was reached. The speed was then increased by $1 \mathrm{~m} \mathrm{~min}^{-1}$ every $60 \mathrm{~s}$ until maximal aerobic velocity. Next, SHR were randomly assigned to sedentary (SHR), moderate (SHR-MOD) or high-intensity exercise (SHR-HI) groups. Exercise sessions consisted of continuous running for $1 \mathrm{~h}, 5$ days a week for 6 weeks at $55 \%$ of maximal aerobic velocity for SHR-MOD and $80 \%$ of maximal aerobic velocity for SHR-HI.

\section{Blood pressure and body mass}

The mean (MBP), systolic (SBP) and diastolic (DBP) blood pressures were assessed in conscious rats by tail-cuff method using the CODA tail-cuff system (Kent Scientific, Torrigton, CT, USA). The rats were warmed for $15 \mathrm{~min}$ before measurements. The rats underwent 3 days of habituation to blood pressure measurements before the baseline blood pressure measurements were assessed. MBP, SBP and DBP were measured at weeks 2, 4 and 6 of the protocol. The MBP, SBP and DBP values were calculated as the average of 10 measurements.

\section{Isolation of aortic rings}

Under anesthesia (sodium pentobarbital, $100 \mathrm{mg} \mathrm{kg}^{-1}$, intraperitoneal), the thoracic aorta was quickly removed and placed in cold Krebs-Henseleit bicarbonate buffer (composition in mM: $\mathrm{NaCl} 118, \mathrm{NaHCO}_{3} 25, \mathrm{KCl} 4.8$, $\mathrm{KH}_{2} \mathrm{PO}_{4} 1.2, \mathrm{CaCl}_{2} 1.25$, glucose 11). After the removal of adherent tissue, the vessels were cut into $2-3-\mathrm{mm}$ long rings. The aortic rings were mounted onto stainless steel supports and suspended in the tissue bath containing Krebs-Henseleit buffer at $37^{\circ} \mathrm{C}$, continuously bubbled with $\mathrm{O}_{2}-\mathrm{CO}_{2}$ $(95 \%-5 \%)$ gas mixture. The rings were connected to an isometric force transducer (EMKA Technologies, EMKA Paris, France), linked to an amplifier (EMKA Technologies) and a computerized acquisition system to record changes in the isometric force. The resting tension was adjusted to $2.0 \mathrm{~g}$. After $60 \mathrm{~min}$ of stabilization, the muscular and endothelial integrity were tested with a single dose of phenylephrine $\left(10^{-6} \mathrm{M}\right)$ and acetylcholine $\left(\mathrm{ACh}, 10^{-6} \mathrm{M}\right)$. Then, each vessel ring was preconstricted with phenylephrine $\left(10^{-6} \mathrm{M}\right)$. After the preconstriction reached a plateau, endothelium-dependent relaxation was examined by challenging the aortic rings with cumulative concentrations of ACh $\left(10^{-9}-3 \cdot 10^{-5} \mathrm{M}\right)$. Considering that eNOS inhibition with L-nitro arginine methyl ester (L-NAME) totally abolishes ACh-dependent vasorelaxation in aortic tissue ${ }^{27}$ which clearly shows that eNOS is the main pathway involved in the endothelium response to $\mathrm{ACh}$ in the aorta, we chose to investigate the eNOS-dependent vasorelaxation in response to ACh by inhibiting the other possible interacting pathways. The eNOS-NO pathway vasorelaxant contribution was therefore evaluated in the presence of a combination of indomethacin $\left(10^{-5} \mathrm{M}\right)$, a cyclooxygenase (COX) inhibitor to blunt the production of $\mathrm{PGI}_{2}$, and apamin $\left(10^{-7} \mathrm{M}\right)$ plus TRAM $34\left(10^{-6} \mathrm{M}\right)$, which are $\mathrm{SK}_{\mathrm{Ca}}$ and $\mathrm{IK}_{\mathrm{Ca}}$ inhibitors, respectively, to blunt the pro-relaxation contribution of endothelium-derived hyperpolarizing factor. All inhibitory drugs were added to the bath $30 \mathrm{~min}$ before the ACh dose-response assay was performed. To evaluate the role of eNOS uncoupling, the contribution of NO pathway in the response of endothelium to ACh was also investigated in the presence of NOS coupling agent $\mathrm{BH}_{4}(100 \mu \mathrm{M})$. Finally, endotheliumindependent relaxation was assessed on preconstricted aortic rings challenged with a maximal dose of sodium nitro-prusside $\left(10^{-5} \mathrm{M}\right)$. The vessel rings were rinsed to stabilize the resting tension levels between each drug intervention.

\section{Western blotting analysis}

Proteins from aorta homogenates were separated on polyacrylamide-sodium dodecyl sulfate gels and transferred onto polyvinylidene difluoride membranes. The membranes were incubated with primary antibodies at $4{ }^{\circ} \mathrm{C}$ in $10 \%$ milk or $3 \%$ bovine serum albumin in Tris-buffered saline containing 0.05\% Tween-20 overnight. The primary antibodies used in this study were anti-mouse eNOS (1:1000; BD transduction Laboratories, Lexington, KY, USA), anti-mouse eNOS-PSer1177 (1:1,000; BD Transduction Laboratory), anti-rabbit GAPDH (glyceraldehyde 3-phosphate dehydrogenase; 1:5000; Santa Cruz Biotechnology, Santa Cruz, CA, USA) and anti-rabbit nitrotyrosine (1:15000; Millipore Corporation, Billerica, MA, USA). Immunodetection was carried out using an ECL or ECL Plus system (SuperSignal West Pico Chemiluminescence Substrate, Thermo Scientific (Villebon-sur-Yvette, France) or Luminata Forte Western HRP substrate, Millipore Corporation, respectively), and the membranes were then exposed to X-ray films for visualization. The eNOS protein content was expressed relative to the GAPDH content. The eNOS-PSer1177 protein content was expressed relative to the eNOS content.

\section{Measurement of ROS}

ROS production was measured by electron paramagnetic resonance in freshly frozen aorta homogenates, as previously described. ${ }^{17}$ Briefly, the homogenates were treated with a solution of CMH (1-hydroxy-3-methoxycarbonyl-2,2,5,5tetramethyl-pyrrolidine) at a concentration of $400 \mu \mathrm{M}(1: 1 \mathrm{v} / \mathrm{v})$, placed into electron paramagnetic resonance glass capillary tubes (Noxygen Science Transfer and Diagnostics, Elzach, Germany) and then placed inside the e-scan spectrometer (Bruker, Ettlingen, Germany) for data acquisition. The procedure was then repeated on the same samples but in the presence of L-NIO $(10 \mu \mathrm{M})$, a specific eNOS inhibitor. The difference between the first and second results was considered to be the eNOS-dependent ROS production. Alternatively, the procedure was also repeated in the presence of $N$-acetylcysteine $(2 \mathrm{mM})$ or of $\mathrm{BH}_{4}(100 \mu \mathrm{M})$. The ROS production was normalized to the protein content of

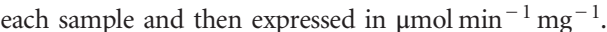

\section{Statistical analysis}

The data are expressed as the mean \pm s.e.m. For comparison of multiple experimental conditions, Student's $t$-test, analysis of variance or repeatedmeasures analysis of variance followed by the Bonferroni adjusted $t$-test were used. A value of $P<0.05$ was considered statistically significant.

\section{RESULTS}

\section{Exercise training does not correct hypertension in SHR}

SHR presented higher SBP, DBP and MBP at the beginning of and throughout the protocol (Figure 1a). At the end of the exercise training, the SBP, DBP and MBP tended to be lower in SHR trained at moderate intensity than in their sedentary counterparts $(5.4,8.9$ and $4.6 \%$, respectively), but the values did not reach significance (Figure 1a). By contrast, high-intensity exercise training did not lead to any changes in SBP, DBP and MBP in SHR (Figure 1a). WKY and SHR had no difference in resting heart rates throughout the protocol 

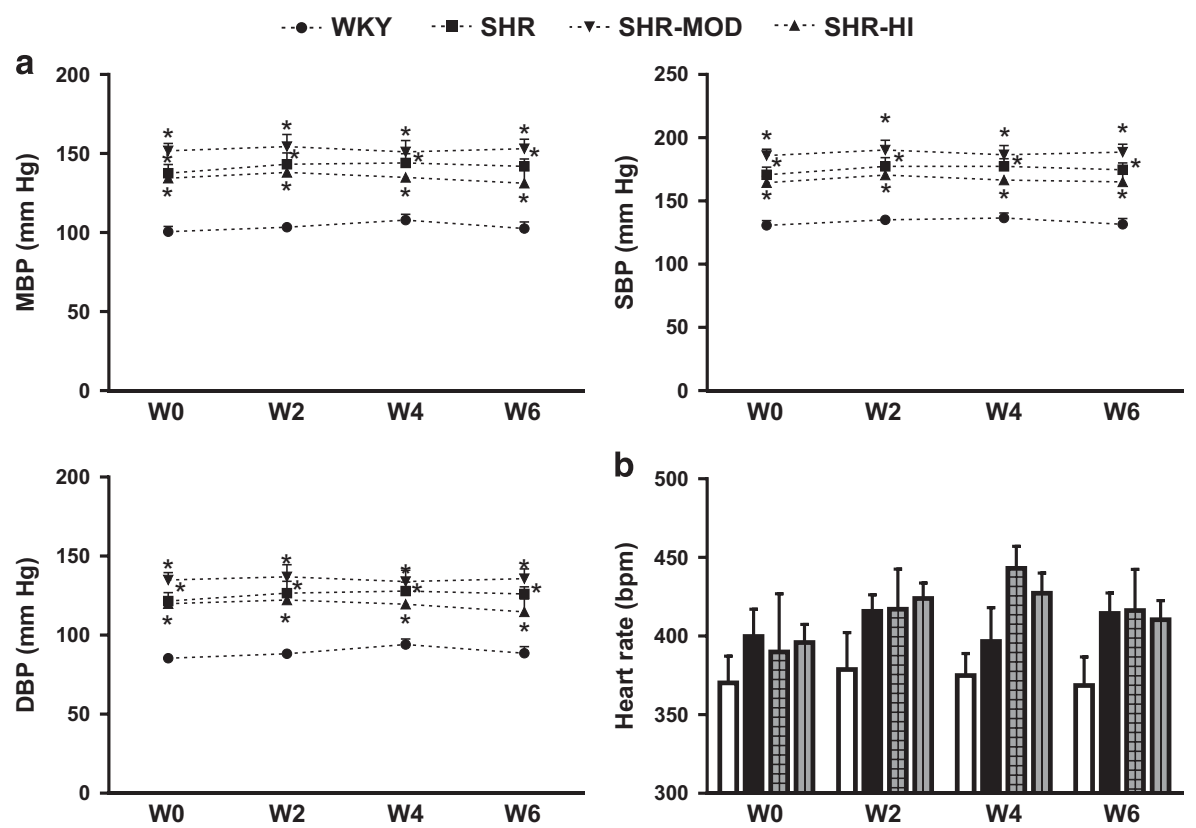

Figure 1 High-intensity exercise training does not correct hypertension in SHR. (a) Evaluation of the mean (MBP), systolic (SBP) and diastolic (DBP) blood pressures at baseline (WO) and in week 2 (W2), week 4 (W4) and week 6 (W6) of the protocol. (b) Evaluation of heart rates during the protocol. Values are the mean \pm s.e.m. ${ }^{*} P<0.05$ vs. WKY.

(Figure 1b). Both moderate- and high-intensity exercise had no effect on this parameter (Figure $1 \mathrm{~b}$ ).

Effect of continuous moderate- or high-intensity exercise training on endothelium-dependent vasorelaxation

The vasorelaxation response to ACh was altered in SHR compared with their normotensive counterparts (Figure 2a). This alteration was characterized by a lower maximal response to ACh (Figure 2c), with no alteration of the sensitivity $\left(\mathrm{EC}_{50}\right)$ (Figure $2 \mathrm{~b}$ ). Considering that no difference was observed regarding the response to sodium nitroprusside (SNP) (WKY, 98.2 $\pm 0.3 \%$; SHR, 98.0 $\pm 1.2 \%$; NS), these results highlight the endothelial dysfunction in the SHR aortas compared with the control rats. Thus we next evaluated whether exercise training was able to modulate endothelium-dependent relaxation in SHR. As expected, in SHR, continuous moderateintensity exercise training improved ACh-dependent vasorelaxation with regard to aortic sensitivity to ACh (Figure 2b) but had no effect on the maximal response (Figure 2c). By contrast, high-intensity exercise training had no significant effect on the maximal response (Figures 2a and c) or the sensitivity (Figure 2b) to Ach but tended to worsen both parameters. No difference in the maximal response to SNP was observed between the WKY, SHR, SHR-MOD and SHR-HI (SHR-MOD, $97.5 \pm 0.6 \%$, SHR-HI, $98.4 \pm 0.7 \%$ ) groups (Figure $2 \mathrm{~d}$ ).

Effect of continuous high-intensity exercise training on eNOS-mediated vasorelaxation

Exercise training is especially known to modulate the eNOS-NO pathway. ${ }^{16,17,19}$ Thus, to better understand the lack of a beneficial effect of high-intensity exercise training on endothelium-dependent vasorelaxation in our model, we next focused our study on the contribution of the eNOS-NO pathway to the endotheliumdependent response to ACh in SHR-HI aortic tissue. Endotheliumdependent vasorelaxation is mainly dependent on (i) NO production by eNOS, (ii) $\mathrm{PGI}_{2}$ synthesis under the control of COX activity and (iii) endothelium-derived hyperpolarizing factor-mediated opening of the $\mathrm{SK}_{\mathrm{Ca}}$ and $\mathrm{IK}_{\mathrm{Ca}}$ channels. To identify the contribution of eNOS to endothelium-dependent vasorelaxation, we performed dose-response experiments with $\mathrm{ACh}$ in the presence of indomethacin, a COX inhibitor, to blunt the production of PGI2, and apamin plus TRAM 34 ( $\mathrm{SK}_{\mathrm{Ca}}$ and $\mathrm{IK}_{\mathrm{Ca}}$ inhibitors, respectively) to blunt the hyperpolarizing contribution to vasorelaxation. We observed that the eNOS-dependent response to ACh in the aorta was not decreased in SHR compared with control rats. Indeed, no alteration of the maximal response to ACh was observed (Figures $3 \mathrm{a}$ and $\mathrm{c}$ ), but a higher sensitivity to ACh was observed in SHR compared with WKY rats (Figure 3b). High-intensity exercise training significantly impaired the eNOS-dependent maximal response to ACh in SHR-HI compared with SHR. In addition, ACh sensitivity, which was increased in SHR, was normalized in SHR-HI to the level observed in the WKY aortas (Figure 3b), suggesting some potential deleterious effect of high-intensity exercise training on the eNOS-NO pathway.

\section{Continuous high-intensity exercise training alters the eNOS} functional state in SHR aortas

Next, to understand these results, we evaluated the effects of our experimental conditions on eNOS and eNOS-Pser1177 by western blotting. The expression of eNOS was increased in SHR aortic tissue with no supplemental effect of exercise training (Figure 4). Indeed, no difference in eNOS expression was observed between SHR and SHR-HI. The eNOS-Pser1177/eNOS ratio was not modified in SHR compared with WKY aortas, and no effect of high-intensity exercise training on this parameter was observed in SHR (Figure 4). Because eNOS produces NO in its coupled state, whereas eNOS uncoupling results in a switch from $\mathrm{NO}$ to $\mathrm{O}_{2}{ }^{-}$generation, ${ }^{28,29}$ we next evaluated ROS production in the aortic tissue in the presence or absence of a specific inhibitor of eNOS (L-NIO, $10 \mu \mathrm{M}$ ). Notably, the total ROS production was increased in both SHR and SHR-HI aortas compared 

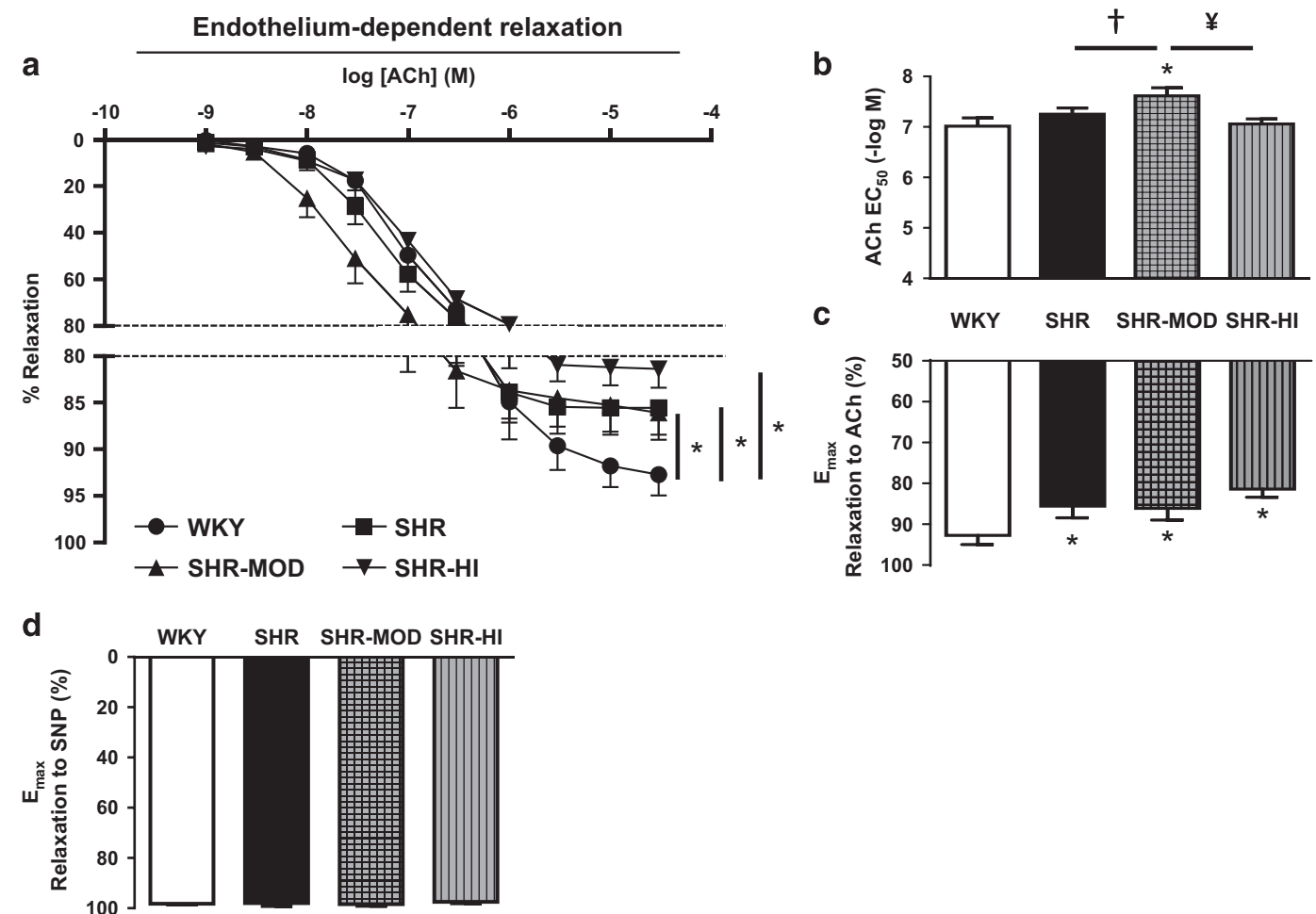

Figure 2 High-intensity exercise training does not correct endothelium dysfunction in SHR. (a) Dose-dependent response of aortic rings to cumulative doses of ACh. The data are expressed as the percentage of relaxation relative to the preconstriction level. (b) ACh concentration inducing $50 \%$ of the maximal response to $\mathrm{ACh}$ in aortic rings. (c) Maximal relaxation of aortic rings in response to Ach expressed as the percentage of relaxation relative to the preconstriction level. (d) Maximal relaxation of aortic rings in response to SNP, expressed as the percentage of relaxation relative to the preconstriction level. The values are the mean \pm s.e.m. ${ }^{*} P<0.05$ vs. WKY group. ${ }^{\dagger} P<0.05$ vs. SHR group. ${ }^{¥} P<0.05$ vs. SHR-MOD.
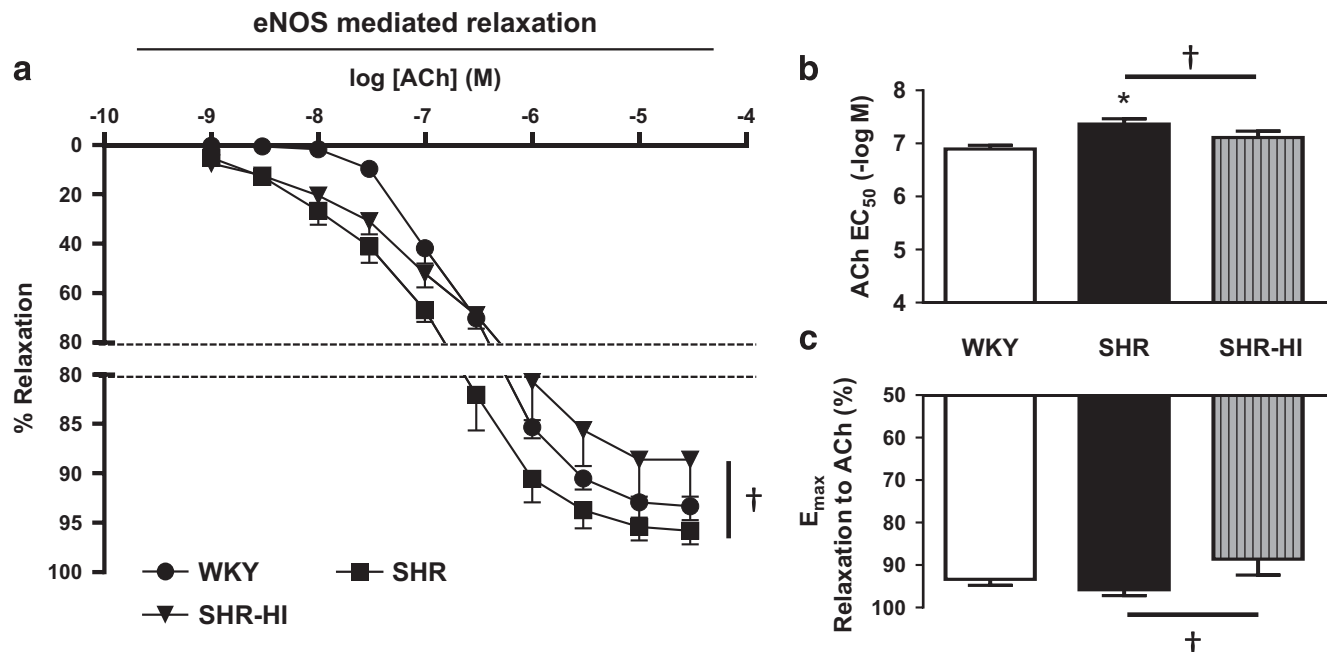

Figure 3 High-intensity exercise decreases eNOS-dependent vasorelaxation in SHR. (a) Dose-dependent response to a cumulative dose of ACh in aortic rings pretreated with indomethacin+TRAM 34+apamin. The data are expressed as the percentage of relaxation relative to the preconstriction level. (b) ACh concentration inducing $50 \%$ of the maximal response to ACh in aortic rings pretreated with indomethacin+TRAM 34+apamin. (c) Maximal relaxation in response to ACh of aortic rings pretreated with indomethacin+TRAM 34+apamin and expressed as the percentage of relaxation relative to the preconstriction level. The values are the mean \pm s.e.m. ${ }^{*} P<0.05$ vs. WKY group. ${ }^{\dagger} P<0.05$ vs. SHR group.

with WKY aortas (Figure 5a). Although no difference in the total aortic ROS production was observed between the SHR and SHR-HI groups, the specific inhibition of eNOS only reduced ROS production in the SHR-HI aortas and had no effect on WKY or SHR (Figure 5a). Thus eNOS-dependent ROS production was higher in the SHR-HI group than in the two other groups (Figure 5b). This result strongly suggests eNOS uncoupling in the SHR-HI aortas. In pro-oxidant conditions, $\mathrm{NO}$ can react with $\mathrm{O}_{2}^{-}$to produce peroxynitrite, which results in a protein modification called nitrotyrosination. Thus, as previously described, ${ }^{17,26}$ we measured aortic nitrotyrosination by 

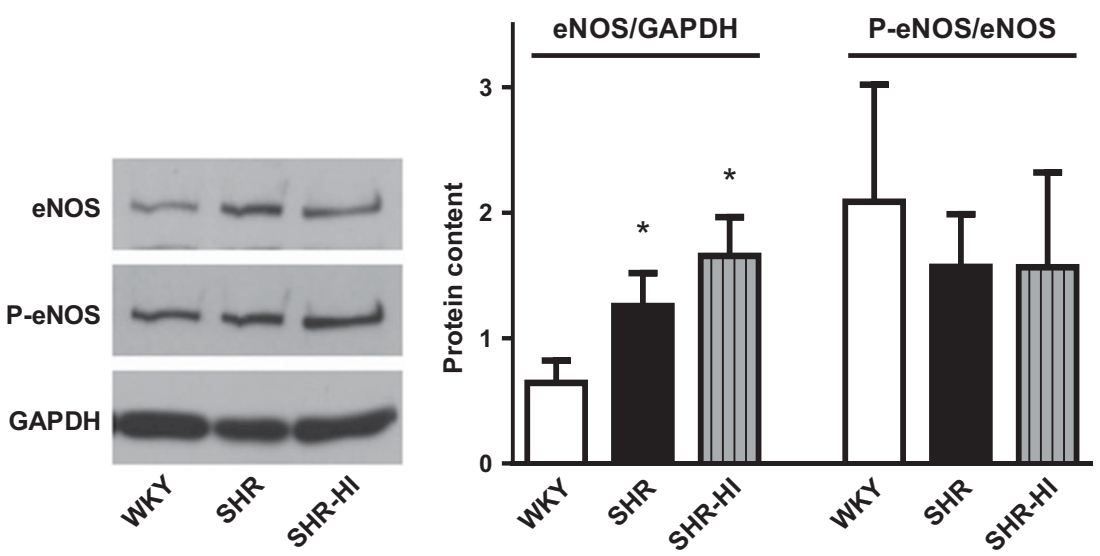

Figure 4 High-intensity exercise does not affect eNOS expression and eNOS phosphorylation in SHR. eNOS expression and phosphorylation at Ser 1177 (P-eNOS) measured by western immunoblotting. The eNOS and P-eNOS levels are relative to the expression values of GAPDH and total eNOS, respectively. The values are the mean \pm s.e.m. ${ }^{*} P<0.05$ vs. WKY group.
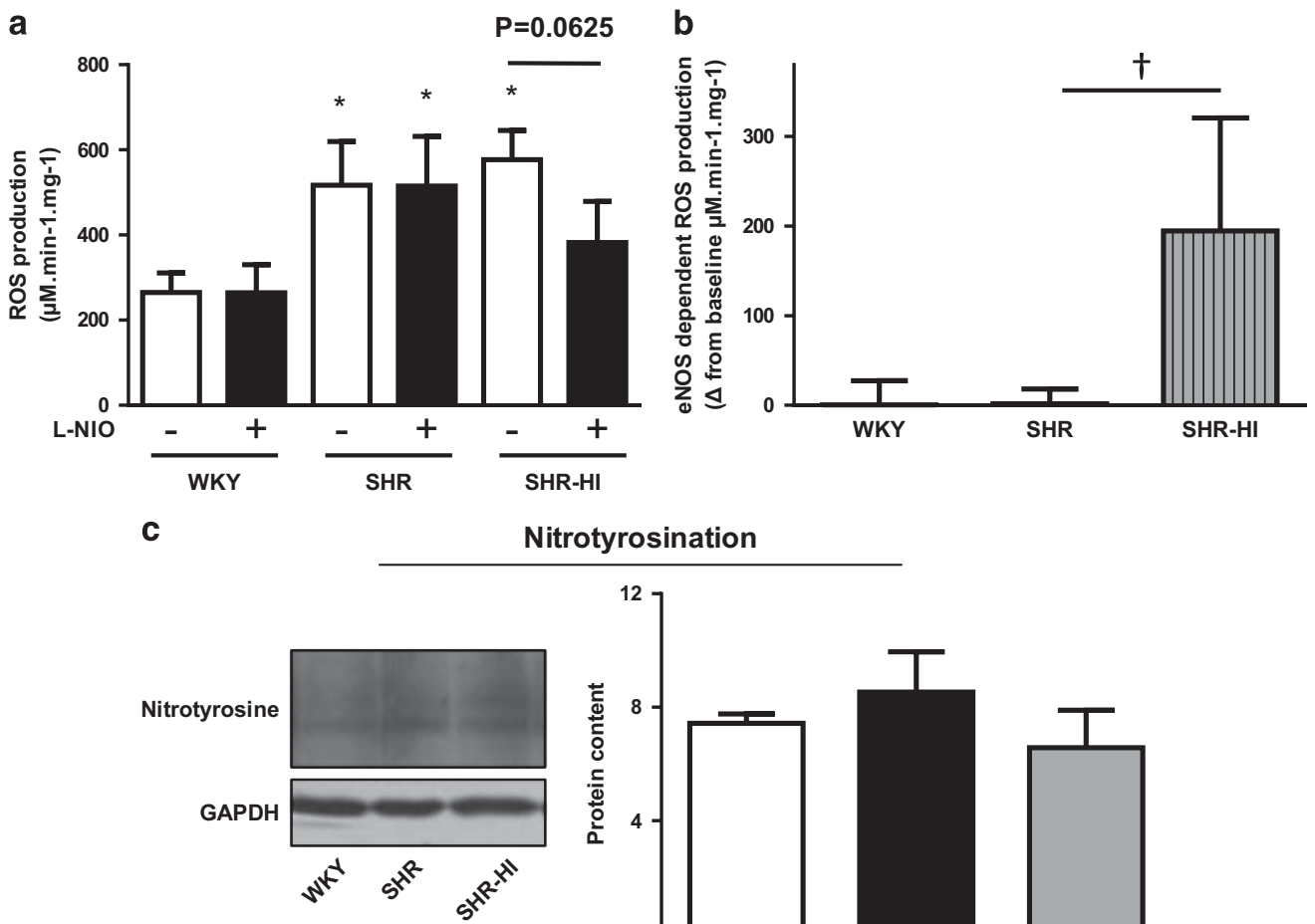

Nitrotyrosination

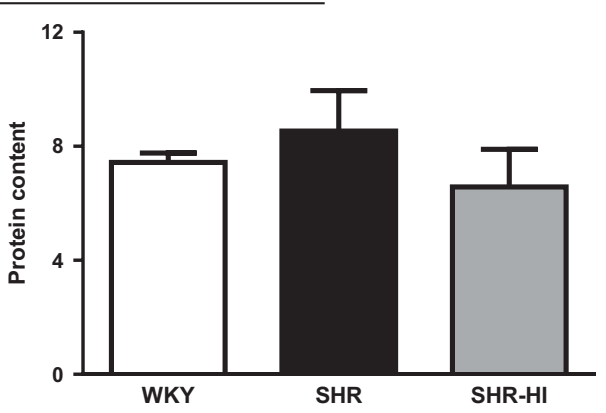

Figure 5 High-intensity exercise increases eNOS uncoupling in SHR. (a) Total aortic ROS production measured by electron paramagnetic resonance (EPR) in fresh frozen aorta homogenates either pretreated with L-NIO or left untreated. (b) eNOS-dependent ROS production measured by EPR in fresh frozen aorta homogenates. The values correspond to the difference between ROS measurements obtained in the presence or absence of L-NIO. (c) Protein nitrotyrosination measured by western immunoblotting. The nitrotyrosination level is relative to the expression values of GAPDH. The values are the mean \pm s.e.m. ${ }^{*} P<0.05$ vs. WKY group. $\dagger P<0.05$ vs. SHR group.

western blotting as an index of peroxynitrite formation. Protein nitrotyrosination tended to be higher in the aortas of SHR, without reaching significance, whereas those from the SHR group that exercised at high intensity tended be less nitrotyrosinated than their sedentary counterparts (Figure $5 \mathrm{c}$ ). Considering that eNOS uncoupling is explained in the literature by either the redox modulation of its S-glutathionylation 28,30 or the oxidation of its co-factor tetrahydrobiopterin $\left(\mathrm{BH}_{4}\right),{ }^{31-33}$ we next evaluated whether the use of a reducing agent $(\mathrm{NAC}, 2 \mathrm{mM})$ or $\mathrm{BH}_{4}(100 \mu \mathrm{m})$ could modulate ROS production in the SHR-HI aortas. Notably, although
NAC had no significant effect on ROS production in the SHR aortas (Figure 6a), the use of this reducing agent significantly blunted ROS production in the SHR-HI aortas (Figure 6a). In addition, the use of the recoupling agent $\mathrm{BH}_{4}$ revealed the same pattern of results; $\mathrm{BH}_{4}$ did not alter ROS production in the aortas of SHR, but it decreased this parameter in the SHR-HI group (Figure 6b). Altogether, these results suggest that high-intensity exercise training is associated with an altered endothelium redox state that subsequently increases the level of eNOS uncoupling in the aortas of SHR. Finally, we measured eNOS-dependent vasorelaxation in both the SHR and SHR-HI aortas 

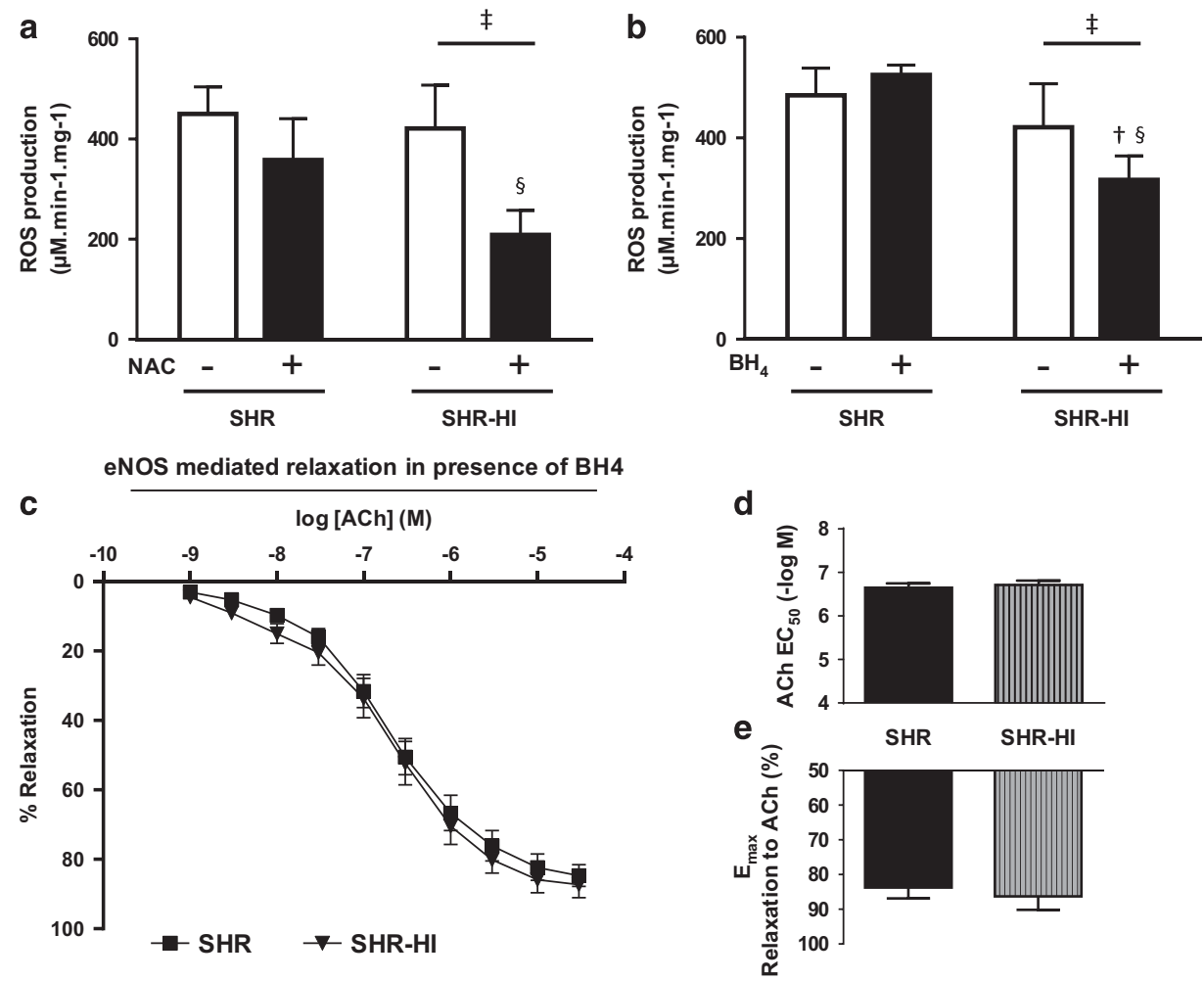

Figure 6 Reducing agent ( $N$-acetylcysteine (NAC)) and $\mathrm{BH}_{4}$ reverse eNOS uncoupling SHR-HI. (a-c) Total aortic ROS production measured by electron paramagnetic resonance (EPR) in freshly frozen aorta homogenates pretreated with NAC (2 mM) or left untreated. (d) Total aortic ROS production measured by EPR in freshly frozen aorta homogenates pretreated with $\mathrm{BH}_{4}(100 \mu \mathrm{m})$ or left untreated. (e) Dose-dependent response to the cumulative addition of ACh on aortic rings pretreated with indomethacin+TRAM 34+apamin and $\mathrm{BH}_{4}$. The data are expressed as the percentage of relaxation relative to the preconstriction level. (f) Half-maximal effective concentration of ACh on aortic rings pretreated with ITA and $\mathrm{BH}_{4}$. (g) Maximal relaxation to ACh in aortic rings pretreated with ITA and $\mathrm{BH}_{4}$. The values are the mean \pm s.e.m. ${ }^{*} P<0.05$ vs. WKY group. ${ }^{\dagger} P<0.05$ vs. SHR group. ${ }^{\ddagger} P<0.05$ vs. SHR-HI group. $\S P<0.05$ vs. SHR treated with $\mathrm{NAC}$ or $\mathrm{BH}_{4}$.

in the presence of $\mathrm{BH}_{4}$. Notably, in the presence of this recoupling agent, no difference between these two populations was observed (Figures 6c-e).

\section{DISCUSSION}

The main finding of this study was that high-intensity exercise training is associated with an altered eNOS functional state in the aortas of SHR, which could explain why this strategy was not able to correct endothelial dysfunction in this population. There is evidence to suggest that the effects of exercise training on the vascular endothelium depend on its intensity. ${ }^{34}$ Indeed, high-intensity exercise may have no positive $\mathrm{e}^{35-37}$ or deleterious effects ${ }^{38,39}$ on endothelial function. Although high-intensity exercise has been consistently reported to have beneficial effects ${ }^{22-24}$ on hypertension in normotensive rats, a previous study by Sun et al. ${ }^{25}$ showed that, in a rat model, low-intensity $(30 \%$ of maximal aerobic velocity) rather than moderate-intensity (60\% of maximal aerobic velocity) exercise conferred beneficial effects on blood pressure and vessel function. Consistent with these results, the present study provides new insight to explain why continuous high-intensity aerobic exercise training is not effective at improving endothelial function in SHR.

The effects of exercise training on endothelial function are well-known consequences of an improved eNOS-NO pathway function. ${ }^{14,40,41}$ However, although the effects of moderate exercise training on this pathway are well described, ${ }^{42-44}$ those of highintensity exercise training are less known and seem controversial. Indeed, several studies have demonstrated that moderate- or low-intensity exercise is effective at increasing NO bioavailability and endothelium-dependent relaxation in both animal models of hypertension ${ }^{11,25,45,46}$ and hypertensive humans ${ }^{38,47,48}$; by contrast, there are only a few studies that have investigated the effect of continuous high-intensity exercise training on endothelial function. Goto et al. ${ }^{43}$ reported that long-term moderate-intensity (50\%

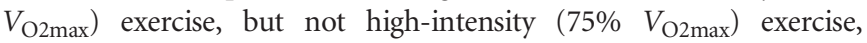
augmented endothelium-dependent vasodilation through increased NO production in healthy subjects. To explain this difference, Goto et al. $^{43}$ suggested that increased oxidative stress (as evidenced by increased plasma concentrations of 8-OHdG (8-hydroxy2 -deoxyguanosine) and serum concentrations of MDA-LDL (malondialdehyde-modified low-density lipoprotein)) may have resulted in decreased NO bioavailability in subjects exercising at high intensity. Similar mechanisms are likely to occur in hypertensive subjects. Indeed, Sun et $a l .{ }^{25}$ compared the effects of low- and high-intensity exercise training in SHR and reported that low-, but not high-intensity, exercise improves endothelium-dependent vasorelaxation. We report here that, although moderate exercise training is well known to increase the level of eNOS phosphorylation at its main activation site in healthy rodents, ${ }^{49,50}$ high-intensity exercise training has no effect on eNOS-Pser1177 in the aortas of SHR. These results could partially explain why no specific effect of high-intensity exercise training was observed on endothelial function, but they are not sufficient to explain why eNOS-dependent vasorelaxation was reduced in SHR-HI compared with their sedentary counterparts. 


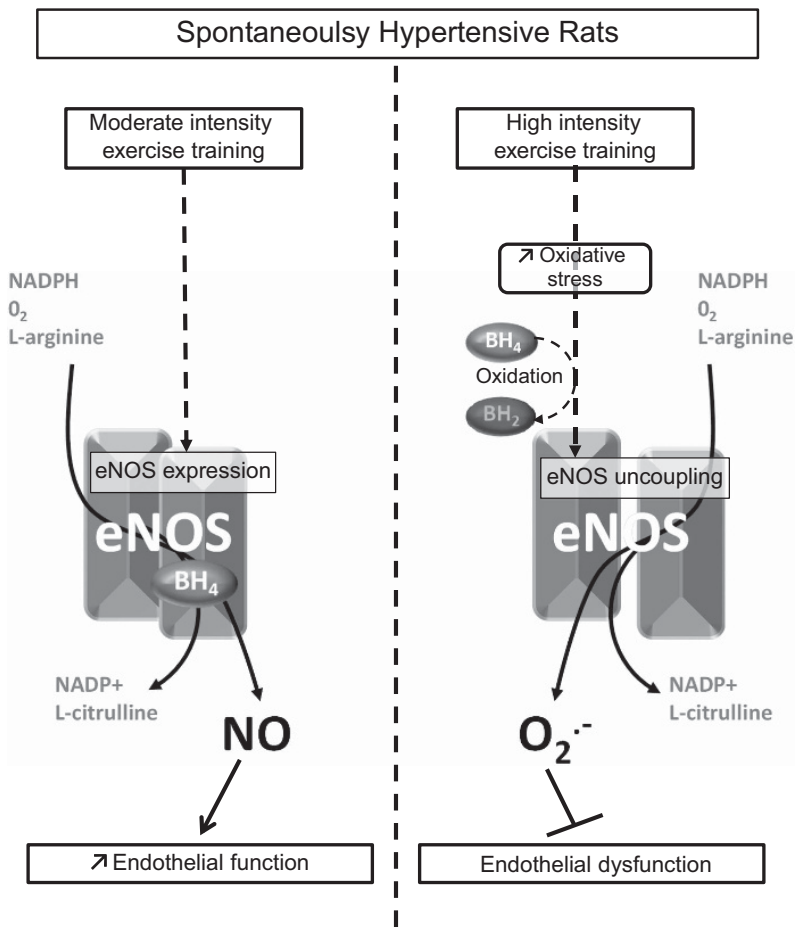

Figure 7 High-intensity exercise does not improve endothelial function in SHR. Moderate-intensity exercise training increases NO bioavailability by increasing the eNOS protein level and therefore improves endothelial function. ${ }^{11,45,46}$ By contrast, high-intensity exercise training that is responsible for increased oxidative stress results in redox-dependent eNOS uncoupling, diminished eNOS/NO function and did not enhance endothelial function.

In pro-oxidant conditions, $\mathrm{NO}$ can react with superoxide anion $\left(\mathrm{O}_{2}^{-}\right)$and produce peroxynitrite, a potent cytotoxic molecule. This biochemical reaction is further associated with the reduced bioavailability of $\mathrm{NO}^{51,52}$ and thus could help to explain our results. However, here, protein nitrotyrosination, an index of peroxynitrite formation, tended to be higher in SHR and was reduced by highintensity exercise training. Because higher eNOS function is associated with higher NO bioavailability and has been reported to be associated with an increased level of protein nitrotyrosination in pro-oxidant conditions, ${ }^{17}$ these results may suggest diminished NO formation in SHR that exercised at high intensity compared with sedentary rats. In addition, high-intensity exercise is well known to be associated with increased oxidative stress when compared with moderate-intensity exercise. ${ }^{53}$ However, in our model, ROS production was increased in SHR, as previously described, ${ }^{54-56}$ and no additional effect of highintensity exercise training was observed. Consequently, the worsening effect of high-intensity exercise on endothelial function could not be directly attributed to oxidative stress.

eNOS is also well known as a critical homodimeric enzyme. In physiological conditions, the dimerized state of the enzyme has a key role in coupling electron transfer in the reductase domain with $\mathrm{NO}$ synthesis in the heme domain via the delivery of electrons required for oxygen activation at the catalytic heme site. However, in some pathological conditions or stress, the enzyme can be uncoupled, resulting in a switch from $\mathrm{NO}$ to $\mathrm{O}_{2}{ }^{-}$synthesis, ${ }^{57-60}$ which is particularly true in hypertension. ${ }^{5,28}$ In our study, although the total ROS production was not different between the SHR and SHR-HI aortas, eNOS-dependent ROS production was increased in the exercised animals, suggesting that, in SHR, high-intensity exercise training was primarily associated with the functional uncoupling of the enzyme. Such uncoupling is mainly explained in the literature by the depletion/oxidation of the cofactor $\mathrm{BH}_{4}^{5,33,61-63}$ or by redox-dependent glutathionylation of the enzyme. ${ }^{28,64}$ In the present study, the use of NAC, an agent with thiol-reducing properties, ${ }^{65-67}$ or $\mathrm{BH}_{4}$, a recoupling agent, ${ }^{68,69}$ reduced ROS production in only SHR-HI aortas, strongly suggesting that this increase in eNOS-dependent ROS production was mainly related to redox-dependent eNOS uncoupling. ${ }^{28}$ Because our data do not suggest increased vascular oxidative stress at the steady state, we can speculate that the eNOS uncoupling observed here is due to the particularly high oxidative stress generated during high-intensity exercise training, ${ }^{53}$ which could result in the oxidation of $\mathrm{BH}_{4}$ and/or the direct redox-dependent glutathionylation of eNOS. Indeed, mitochondrial ROS production increased with regard to oxygen consumption. ${ }^{70}$ However, the ability of chronic treatment with $\mathrm{BH}_{4}$ to prevent the deleterious effects of high-intensity training should be tested to better evaluate this point. Finally, because no difference was reported in eNOS-dependent vasorelaxation between the SHR and SHR-HI aortic rings in the presence of $\mathrm{BH}_{4}$, we can conclude that the eNOS uncoupling consequence of continuous high-intensity exercise training mainly helps to explain why this strategy has no beneficial effect on arterial endothelial function in SHR.

\section{Study limitation}

In the present study, data obtained on the functional state of eNOS are mainly focused on the impact of high-intensity exercise training, and no data are provided on how moderate exercise training could affect this pathway. However, even if this could constitute a limitation of the present work, the effect of moderate exercise training on the eNOS-NO pathway has already been studied not only in healthy ${ }^{37,71}$ but also in SHR. ${ }^{11,45,46}$ Those studies reported that exercise training at moderate intensity improve the eNOS-NO pathway function.

\section{CONCLUSION}

In conclusion, we report here that continuous high-intensity exercise training is associated with the dysfunction of the eNOS-NO pathway, characterized mainly by a lack of increase in eNOS phosphorylation of the enzyme at its activation site and by its functional uncoupling, thus increasing eNOS-dependent ROS production (Figure 7). These effects of continuous high-intensity exercise training on the functional state of eNOS could help to explain why this exercise training strategy cannot improve endothelial function in SHR and therefore cannot be considered a good protective strategy in this population.

\section{CONFLICT OF INTEREST}

The authors declare no conflict of interest.

\section{ACKNOWLEDGEMENTS}

S Battault's salary was supported by Laboratoire-Lescuyer.

1 Kearney PM, Whelton M, Reynolds K, Muntner P, Whelton PK, He J. Global burden of hypertension: analysis of worldwide data. Lancet 2005; 365: 217-223.

2 Ghiadoni L, Taddei S, Virdis A. Hypertension and endothelial dysfunction: therapeutic approach. Curr Vasc Pharmacol 2012; 10: 42-60.

3 Shantsila A, Shantsila E. Arterial stiffening in hypertension: beyond blood pressure levels. J Hum Hypertens 2010; 24: 303-305.

4 Forte P, Copland M, Smith LM, Milne E, Sutherland J, Benjamin N. Basal nitric oxide synthesis in essential hypertension. Lancet 1997; 349: 837-842. 
5 Landmesser U, Dikalov S, Price SR, McCann L, Fukai T, Holland SM, Mitch WE, Harrison DG. Oxidation of tetrahydrobiopterin leads to uncoupling of endothelial cel nitric oxide synthase in hypertension. J Clin Invest 2003; 111: 1201-1209.

6 Panza JA, Casino PR, Kilcoyne CM, Quyyumi AA. Role of endothelium-derived nitric oxide in the abnormal endothelium-dependent vascular relaxation of patients with essential hypertension. Circulation 1993; 87: 1468-1474.

7 Panza JA, García CE, Kilcoyne CM, Quyyumi AA, Cannon RO. Impaired endotheliumdependent vasodilation in patients with essential hypertension. Evidence that nitric oxide abnormality is not localized to a single signal transduction pathway. Circulation 1995; 91: 1732-1738.

8 Schiffrin EL, Park JB, Intengan HD, Touyz RM. Correction of arterial structure and endothelial dysfunction in human essential hypertension by the angiotensin receptor antagonist losartan. Circulation 2000; 101: 1653-1659.

9 Modena MG, Bonetti L, Coppi F, Bursi F, Rossi R. Prognostic role of reversible endothelial dysfunction in hypertensive postmenopausal women. J Am Coll Cardiol 2002; 40: 505-510.

10 Beck DT, Martin JS, Casey DP, Braith RW. Exercise training improves endothelia function in resistance arteries of young prehypertensives. J Hum Hypertens 2014; 28: 303-309.

11 Graham DA, Rush JWE. Exercise training improves aortic endothelium-dependent vasorelaxation and determinants of nitric oxide bioavailability in spontaneously hypertensive rats. J Appl Physiol 2004; 96: 2088-2096.

12 Green DJ, Maiorana A, O'Driscoll G, Taylor R. Effect of exercise training on endothelium-derived nitric oxide function in humans. J Physiol 2004; 561: 1-25.

13 Walsh JH, Bilsborough W, Maiorana A, Best M, O'Driscoll GJ, Taylor RR, Green DJ. Exercise training improves conduit vessel function in patients with coronary artery disease. J Appl Physiol 2003; 95: 20-25.

14 Davis ME, Cai H, McCann L, Fukai T, Harrison DG. Role of $\mathrm{c}-\mathrm{Src}$ in regulation of endothelial nitric oxide synthase expression during exercise training. Am J Physiol Heart Circ Physiol 2003; 284: H1449-H1453.

15 Indolfi C, Torella D, Coppola C, Curcio A, Rodriguez F, Bilancio A, Leccia A, Arcucci O, Falco M, Leosco D, Chiariello M. Physical training increases enos vascular expression and activity and reduces restenosis after balloon angioplasty or arterial stenting in rats. Circ Res 2002; 91: 1190-1197.

16 Hambrecht R, Adams V, Erbs S, Linke A, Kränkel N, Shu Y, Baither Y, Gielen S, Thiele H, Gummert JF, Mohr FW, Schuler G. Regular physical activity improves endothelial function in patients with coronary artery disease by increasing phosphorylation of endothelial nitric oxide synthase. Circulation 2003; 107: 3152-3158.

17 Farah C, Kleindienst A, Bolea G, Meyer G, Gayrard S, Geny B, Obert P, Cazorla O, Tanguy S, Reboul C. Exercise-induced cardioprotection: a role for eNOS uncoupling and NO metabolites. Basic Res Cardiol 2013; 108: 389.

18 Grijalva J, Hicks S, Zhao X, Medikayala S, Kaminski PM, Wolin MS, Edwards JG. Exercise training enhanced myocardial endothelial nitric oxide synthase (eNOS) function in diabetic Goto-Kakizaki (GK) rats. Cardiovasc Diabetol 2008; 7: 34.

19 Gielen S, Schuler G, Adams V. Cardiovascular effects of exercise training molecular mechanisms. Circulation 2010; 122: 1221-1238.

20 Gielen S, Schuler G, Hambrecht R. Exercise training in coronary artery disease and coronary vasomotion. Circulation 2001; 103: e1-e6

21 Linke A. Effects of exercise training upon endothelial function in patients with cardiovascular disease. Front Biosci 2008; 13: 424.

22 Reboul C, Tanguy S, Dauzat M, Obert P. Altitude negates the benefits of aerobic training on the vascular adaptations in rats. Med Sci Sports Exerc 2005; 37: 979-985.

23 Delp MD, McAllister RM, Laughlin MH. Exercise training alters endothelium-dependent vasoreactivity of rat abdominal aorta. J Appl Physiol (1985) 1993; 75: 1354-1363.

24 Delp MD, Laughlin MH. Time course of enhanced endothelium-mediated dilation in aorta of trained rats. Med Sci Sports Exerc 1997; 29: 1454-1461.

25 Sun M-W, Qian F-L, Wang J, Tao T, Guo J, Wang L, Lu A-Y, Chen H. Low-intensity voluntary running lowers blood pressure with simultaneous improvement in endothelium-dependent vasodilatation and insulin sensitivity in aged spontaneously hypertensive rats. Hypertens Res 2008; 31: 543-552.

26 Meyer G, André L, Kleindienst A, Singh F, Tanguy S, Richard S, Obert P, Boucher F, Jover B, Cazorla O, Reboul C. Carbon monoxide increases inducible NOS expression that mediates $\mathrm{CO}$-induced myocardial damage during ischemia-reperfusion. $\mathrm{Am} J$ Physiol Heart Circ Physiol 2015; 308: H759-H767.

27 Loria AS, Brinson KN, Fox BM, Sullivan JC. Sex-specific alterations in NOS regulation of vascular function in aorta and mesenteric arteries from spontaneously hypertensive rats compared to Wistar Kyoto rats. Physiol Rep 2014; 2: e12125.

28 Chen C-A, Wang T-Y, Varadharaj S, Reyes LA, Hemann C, Talukder MAH, Chen Y-R, Druhan LJ, Zweier JL. S-glutathionylation uncouples eNOS and regulates its cellular and vascular function. Nature 2010; 468: 1115-1118.

29 Förstermann U, Münzel T. Endothelial nitric oxide synthase in vascular disease from marvel to menace. Circulation 2006; 113: 1708-1714.

30 Dalle-Donne I, Rossi R, Colombo G, Giustarini D, Milzani A. Protein S-glutathionylation: a regulatory device from bacteria to humans. Trends Biochem Sci 2009; 34: 85-96.

31 Bendall JK, Douglas G, McNeill E, Channon KM, Crabtree MJ. Tetrahydrobiopterin in cardiovascular health and disease. Antioxid Redox Signal 2014; 20: 3040-3077.

32 Crabtree MJ, Hale AB, Channon KM. Dihydrofolate reductase protects endothelial nitric oxide synthase from uncoupling in tetrahydrobiopterin deficiency. Free Radic Biol Med 2011; 50: 1639-1646.

33 Setoguchi S, Hirooka Y, Eshima K, Shimokawa H, Takeshita A. Tetrahydrobiopterin improves impaired endothelium-dependent forearm vasodilation in patients with heart failure. J Cardiovasc Pharmacol 2002 : 363-368.
34 Kemi OJ, Haram PM, Loennechen JP, Osnes J-B, Skomedal T, Wisløff U, Ellingsen $\varnothing$. Moderate vs. high exercise intensity: differential effects on aerobic fitness, cardiomyocyte contractility, and endothelial function. Cardiovasc Res 2005; 67: 161-172.

35 Bergholm R, Mäkimattila S, Valkonen M, Liu ML, Lahdenperä S, Taskinen MR, Sovijärvi A, Malmberg $\mathrm{P}$, Yki-Järvinen $\mathrm{H}$. Intense physical training decreases circulating antioxidants and endothelium-dependent vasodilatation in vivo. Atherosclerosis 1999; 145: 341-349.

36 Johnson JL, Slentz CA, Houmard JA, Samsa GP, Duscha BD, Aiken LB, McCartney JS, Tanner CJ, Kraus WE. Exercise training amount and intensity effects on metabolic syndrome (from studies of a targeted risk reduction intervention through defined exercise). Am J Cardiol 2007; 100: 1759-1766.

37 Sun M-W, Zhong M-F, Gu J, Qian F-L, Gu J-Z, Chen H. Effects of different levels of exercise volume on endothelium-dependent vasodilation: roles of nitric oxide synthase and heme oxygenase. Hypertens Res 2008; 31: 805-816.

38 Roberts CK, Vaziri ND, Barnard RJ. Effect of diet and exercise intervention on blood pressure, insulin, oxidative stress, and nitric oxide availability. Circulation 2002; 106 2530-2532.

39 Doonan RJ, Mutter A, Egiziano G, Gomez Y-H, Daskalopoulou SS. Differences in arterial stiffness at rest and after acute exercise between young men and women. Hypertens Res 2013; 36: 226-231.

40 Sheldon RD, Laughlin MH, Rector RS. Reduced hepatic eNOS phosphorylation is associated with NAFLD and type 2 diabetes progression and is prevented by daily exercise in hyperphagic OLETF rats. J Appl Physiol (1985) 2014; 116: 1156-1164.

41 Li Q-X, Xiong Z-Y, Hu B-P, Tian Z-J, Zhang H-F, Gou W-Y, Wang H-C, Gao F, Zhang Q-J. Aging-associated insulin resistance predisposes to hypertension and its reversal by exercise: the role of vascular vasorelaxation to insulin. Basic Res Cardiol 2009; 104: 269-284.

42 Wang Y, Wang S, Wier WG, Zhang Q, Jiang H, Li Q, Chen S, Tian Z, Li Y, Yu X, Zhao M, Liu J, Yang J, Zhang J, Zang W. Exercise improves the dilatation function of mesenteric arteries in postmyocardial infarction rats via a PI3K/Akt/eNOS pathway-mediated mechanism. Am J Physiol Heart Circ Physiol 2010; 299: H2097-H2106.

43 Goto C, Higashi Y, Kimura M, Noma K, Hara K, Nakagawa K, Kawamura M, Chayama K, Yoshizumi M, Nara I. Effect of different intensities of exercise on endotheliumdependent vasodilation in humans role of endothelium-dependent nitric oxide and oxidative stress. Circulation 2003; 108: 530-535.

44 Higashi Y, Yoshizumi M. Exercise and endothelial function: role of endothelium-derived nitric oxide and oxidative stress in healthy subjects and hypertensive patients. Pharmacol Ther 2004; 102: 87-96.

45 Yen MH, Yang JH, Sheu JR, Lee YM, Ding YA. Chronic exercise enhances endothelium-mediated dilation in spontaneously hypertensive rats. Life Sci 1995; 57: 2205-2213.

46 Chen $\mathrm{Hi} \mathrm{H}-$., Chiang I-P, Jen CJ. Exercise Training Increases Acetylcholine-Stimulated Endothelium-Derived Nitric Oxide Release in Spontaneously Hypertensive Rats. J Biomed Sci 1996; 3: 454-460.

47 Khalid T, Nesreen E, Ramadhan O. Effects of exercise training on postmenopausal hypertension: implications on nitric oxide levels. Med J Malaysia 2013; 68: 459-464.

48 Higashi Y, Sasaki S, Kurisu S, Yoshimizu A, Sasaki N, Matsuura H, Kajiyama G, Oshima T. Regular Aerobic Exercise Augments Endothelium-Dependent Vascular Relaxation In Normotensive As Well As Hypertensive Subjects Role Of EndotheliumDerived Nitric Oxide. Circulation 1999; 100: 1194-1202.

49 Tanaka LY, Bechara LRG, Santos AM, dos, Jordão CP, de Sousa LGO, Bartholomeu T, Ventura LI, Laurindo FRM, Ramires PR. Exercise improves endothelial function: a local analysis of production of nitric oxide and reactive oxygen species. Nitric Oxide Biol Chem 2015; 45: 7-14.

50 Zhang Q-J, McMillin SL, Tanner JM, Palionyte M, Abel ED, Symons JD. Endothelial nitric oxide synthase phosphorylation in treadmill-running mice: role of vascular signalling kinases. J Physiol 2009; 587: 3911-3920.

51 Landmesser U, Harrison DG, Drexler H. Oxidant stress-a major cause of reduced endothelial nitric oxide availability in cardiovascular disease. Eur J Clin Pharmacol 2006; 62: 13-19.

52 Hirooka Y, Eshima K, Setoguchi S, Kishi T, Egashira K, Takeshita A. Vitamin C improves attenuated angiotensin ii-induced endothelium-dependent vasodilation in human forearm vessels. Hypertens Res 2003; 26: 953-959.

53 Alessio HM, Goldfarb AH, Cutler RG. MDA content increases in fast- and slow-twitch skeletal muscle with intensity of exercise in a rat. Am J Physiol 1988; 255: C874-C877

54 Cosentino F, Patton S, Uscio LV d', Werner ER, Werner-Felmayer G, Moreau P, Malinski T, Lüscher TF. Tetrahydrobiopterin alters superoxide and nitric oxide release in prehypertensive rats. J Clin Invest 1998; 101: 1530-1537.

55 Tschudi MR, Mesaros S, Lüscher TF, Malinski T. Direct in situ measurement of nitric oxide in mesenteric resistance arteries increased decomposition by superoxide. Hypertension 1996; 27: 32-35.

56 Zalba G, Beaumont FJ, José GS, Fortuño A, Fortuño MA, Etayo JC, Díez J. Vascular $\mathrm{NADH} / \mathrm{NADPH}$ oxidase is involved in enhanced superoxide production in spontaneously hypertensive rats. Hypertension 2000; 35: 1055-1061.

57 Thum T, Fraccarollo D, Schultheiss M, Froese S, Galuppo P, Widder JD, Tsikas D, Ertl G, Bauersachs J. Endothelial nitric oxide synthase uncoupling impairs endothelial progenitor cell mobilization and function. Diabetes 2007; 56: 666-674.

58 De Pascali F, Hemann C, Samons K, Chen C-A, Zweier JL. Hypoxia and reoxygenation induce endothelial nitric oxide synthase uncoupling in endothelial cells through tetrahydrobiopterin depletion and S-glutathionylation. Biochemistry (Mosc) 2014; 53: 3679-3688. 
59 Marchesi C, Ebrahimian T, Angulo O, Paradis P, Schiffrin EL. Endothelial nitric oxide synthase uncoupling and perivascular adipose oxidative stress and inflammation contribute to vascular dysfunction in a rodent model of metabolic syndrome. Hypertension 2009; 54: 1384-1392.

60 Leggio M, Mazza A, Cruciani G, Sgorbini L, Pugliese M, Bendini MG, Severi P, Jesi AP. Effects of exercise training on systo-diastolic ventricular dysfunction in patients with hypertension: an echocardiographic study with tissue velocity and strain imaging evaluation. Hypertens Res 2014; 37: 649-654.

61 Vásquez-Vivar J, Kalyanaraman B, Martásek P. The role of tetrahydrobiopterin in superoxide generation from eNOS: enzymology and physiological implications. Free Radic Res 2003; 37: 121-127.

62 Wang S, Xu J, Song P, Wu Y, Zhang J, Choi HC, Zou M-H. Acute inhibition of guanosine triphosphate cyclohydrolase 1 uncouples endothelial nitric oxide synthase and elevates blood pressure. Hypertension 2008; 52: 484-490.

63 Heffernan KS, Yoon ES, Sharman JE, Davies JE, Shih Y-T, Chen C-H, Fernhall B, Jae SY. Resistance exercise training reduces arterial reservoir pressure in older adults with prehypertension and hypertension. Hypertens Res 2013; 36: 422-427.

64 Crabtree MJ, Brixey R, Batchelor H, Hale AB, Channon KM. Integrated redox sensor and effector functions for tetrahydrobiopterin- and glutathionylation-dependent endothelial nitric-oxide synthase uncoupling. J Biol Chem 2013; 288: 561-569.
65 Konarkowska B, Aitken JF, Kistler J, Zhang S, Cooper GJS. Thiol reducing compounds prevent human amylin-evoked cytotoxicity. FEBS J 2005; 272: 4949-4959.

66 Laragione T, Bonetto V, Casoni F, Massignan T, Bianchi G, Gianazza E, Ghezzi P. Redox regulation of surface protein thiols: identification of integrin alpha-4 as a molecular target by using redox proteomics. Proc Natl Acad Sci USA 2003; 100: 14737-14741.

67 Gurovich AN, Braith RW. Enhanced external counterpulsation creates acute blood flow patterns responsible for improved flow-mediated dilation in humans. Hypertens Res 2013; 36: 297-305.

68 Bevers LM, Braam B, Post JA, van Zonneveld AJ, Rabelink TJ, Koomans HA, Verhaar MC, Joles JA. Tetrahydrobiopterin, but not L-arginine, decreases NO synthase uncoupling in cells expressing high levels of endothelial NO synthase. Hypertension 2006; 47: 87-94.

69 Cai S, Khoo J, Channon KM. Augmented $\mathrm{BH} 4$ by gene transfer restores nitric oxide synthase function in hyperglycemic human endothelial cells. Cardiovasc Res 2005; 65: 823-831.

70 Powers SK, Jackson MJ. Exercise-induced oxidative stress: cellular mechanisms and impact on muscle force production. Physiol Rev 2008; 88: 1243-1276.

71 Sindler AL, Delp MD, Reyes R, Wu G, Muller-Delp JM. Effects of ageing and exercise training on eNOS uncoupling in skeletal muscle resistance arterioles. J Physiol 2009; 587: 3885-3897. 